\title{
Predictors of massive haemoptysis after a first episode of mild-to-moderate haemoptysis in patients with cystic fibrosis
}

\author{
To the Editor:
}

The main cause of death of cystic fibrosis (CF) patients is respiratory disease due to secondary infections, haemoptysis, pneumothorax, and respiratory failure [1]. In a study of 129 French CF patients who died between 2007 and 2010 without receiving lung transplantation, $8.5 \%$ of deaths were due to haemoptysis [2]. Factors associated with an increased risk of haemoptysis in CF patients include older age, advanced lung disease (forced expiratory volume in $1 \mathrm{~s}\left(\mathrm{FEV}_{1}\right)<70 \%$ predicted), airway colonisation by Pseudomonas aeruginosa [3], CF-related diabetes, portal hypertension, and liver cirrhosis [4]. To our knowledge, there have been no studies of the risk factors for the development of massive haemoptysis $(\mathrm{MH})$ in $\mathrm{CF}$ patients who have previously experienced an episode of mild-to-moderate haemoptysis.

We retrospectively analysed data from all adult CF patients seen at the Lille CF Centre between 2008 and 2018 who had a history of at least one mild-to-moderate haemoptysis episode. All individuals gave written informed consent. Approval for the use of these data was provided by the Institutional Review Board of the French Learned Society for Pulmonology (CEPRO 2015-004). The patients were followed up from the time of mild-to-moderate haemoptysis (or from the first episode for those with $>1$ mild-to-moderate haemoptysis) until they experienced an MH event ("with MH" group) or until the end of follow-up ("without MH" group). Clinicopathological data were collected from medical records. Mild-to-moderate haemoptysis was defined as an episode resulting in 5-240 $\mathrm{mL}$ externalised blood. $\mathrm{MH}$ was defined as: 1) $>240 \mathrm{~mL}$ externalised blood in $24 \mathrm{~h}, 2)>100 \mathrm{~mL}$ externalised blood over $\geqslant 2$ days [5], or 3) any externalised blood loss causing abnormal gas exchange/airway obstruction or haemodynamic instability [6]. Haemoptysis was treated according to the $\mathrm{CF}$ Foundation Consensus Conference Report [7], and patients with $\mathrm{MH}$ received concomitant therapy with intravenous (i.v.) antibiotics. Long-term drug therapy was defined as medication prescribed for $>3$ months. The cumulative incidence of MH was estimated using the Kaplan-Meier method by censoring patients lost to follow-up at the last available visit. The median follow-up time from first mild-to-moderate haemoptysis episode was calculated using the reverse Kaplan-Meier method. Associations between baseline characteristics and $\mathrm{MH}$ occurrence were analysed using univariable Cox proportional hazard regression models. The $\log$-linearity assumption for continuous factors was checked by examining Martingale residual plots and using restricted cubic spline functions, and the proportional hazard assumption for each potential predictive factor was checked by examining the Schoenfeld residuals. Mild-to-moderate haemoptysis recurrence rates were calculated as the number of episodes per 100 person-months from the time of first mild-to-moderate haemoptysis episode to $\mathrm{MH}$ or, for patients without $\mathrm{MH}$, to the last annual review. All statistical tests were performed at a two-tailed $\alpha$ level of 0.05 .

A total of $85 \mathrm{CF}$ patients experienced at least one episode of mild-to-moderate haemoptysis; of these, 23 were excluded for missing data (no follow-up after the episode of mild-to-moderate haemoptysis, transfer to

@ERSpublications

Mild-to-moderate haemoptysis $(\mathrm{m}-\mathrm{mH})$ is common in patients with cystic fibrosis but the risk of subsequent massive haemoptysis (MH) is not known. Allergic bronchopulmonary aspergillosis and diabetes were significant predictors of $\mathrm{MH}$ subsequent to $\mathrm{m}-\mathrm{mH}$. https://bit.ly/ $30093 \mathrm{Hw}$

Cite this article as: Pavaut G, Kyheng M, Le Rouzic O, et al. Predictors of massive haemoptysis after a first episode of mild-to-moderate haemoptysis in patients with cystic fibrosis. ERJ Open Res 2020; 6: 00382-2020 [https://doi.org/10.1183/23120541.00382-2020]. 
another unit, lung transplant, or otherwise not indicated) and the remaining 62 patients were included in the study. 15 (24.2\%) of the 62 patients experienced MH during follow-up (median 73 months, interquartile range 37-132 months). Baseline characteristics obtained at the most recent annual review after mild-to-moderate haemoptysis (when the patients were stable) are shown in table 1. Univariate analysis indicated that allergic bronchopulmonary aspergillosis (ABPA), CF-related diabetes and treatment with triazoles were significantly associated with subsequent $\mathrm{MH}$. Positive and negative association trends were detected between $\mathrm{MH}$ and severe malnutrition and body mass index (BMI), respectively, but they did not reach the level of significance. Interestingly, $\mathrm{MH}$ occurred in four of the five patients with liver cirrhosis and three of the four patients with thrombocytopenia (including two patients with liver cirrhosis) at 24-55 months and 24-103 months, respectively, after the first mild-to-moderate haemoptysis episode. However, the small number of patients with these conditions precluded statistical analysis of possible associations. Mild-to-moderate haemoptysis recurrence was more common among patients who did versus those who did not experience $\mathrm{MH}$ (mild-to-moderate haemoptysis rate 16.5 and 5.9 per 100 patient-months, respectively). $\mathrm{MH}$ was not significantly associated with any other baseline characteristic. Among the 15 patients who had $\mathrm{MH}, 11$ $(73.3 \%)$ were treated with terlipressin, $11(73.3 \%)$ underwent bronchial artery embolisation (BAE), and nine $(60 \%)$ received both treatments. Despite appropriate management, three $(20 \%)$ of the 15 patients died from the $\mathrm{MH}$ event. There were no deaths among patients who did not experience $\mathrm{MH}$.

Thompson et al. [3] reported that haemoptysis is more common in CF individuals with advanced lung disease $\left(\mathrm{FEV}_{1}<70 \%\right.$ predicted). Although we did not detect an association between $\mathrm{FEV}_{1}$ and $\mathrm{MH}$ in our

TABLE 1 Univariate analysis of the association between baseline characteristics and massive haemoptysis (MH) occurrence in patients with cystic fibrosis

\begin{tabular}{|c|c|c|c|c|}
\hline Characteristic & Patients without MH & Patients with $\mathrm{MH}$ & Hazard ratio $(95 \% \mathrm{CI})$ & p-value \\
\hline Subjects $n$ & 47 & 15 & & \\
\hline Age years median (IQR) & $22.0(20.5-30.0)$ & $19.0(16.0-22.0)$ & $1.01(0.96-1.04)$ & 0.93 \\
\hline Male & $28(59.6 \%)$ & $6(40.0 \%)$ & $0.97(0.33-2.85)$ & 0.96 \\
\hline Homozygous $\Delta \mathrm{F} 508 / \Delta \mathrm{F} 508$ & $18(38.3 \%)$ & $6(40.0 \%)$ & $1.04(0.36-3.01)$ & 0.94 \\
\hline Heterozygous $\Delta \mathrm{F} 508$ & $18(38.3 \%)$ & $5(33.3 \%)$ & $1.62(0.53-4.97)$ & 0.40 \\
\hline Body mass index $\mathrm{kg} \cdot \mathrm{m}^{-2}$ & $20.1 \pm 2.2$ & $18.5 \pm 2.75$ & $0.79(0.61-1.02)$ & 0.075 \\
\hline Severe malnutrition & $8(17.0 \%)$ & $7(46.7 \%)$ & $2.69(0.94-7.68)$ & 0.064 \\
\hline FEV $_{1} \%$ predicted & $57.3 \pm 24.6$ & $71.9 \pm 19.8$ & $1.01(0.98-1.03)$ & 0.55 \\
\hline FVC $\%$ predicted & $74.8 \pm 20.6$ & $79.7 \pm 19.6$ & $0.99(0.96-1.03)$ & 0.69 \\
\hline EPI & $41(87.2 \%)$ & $12(80.0 \%)$ & $1.12(0.25-5.04)$ & 0.88 \\
\hline ABPA & $12(25.5 \%)$ & $9(60.0 \%)$ & 6.03 (1.97-18.45) & 0.002 \\
\hline Liver cirrhosis & $1(2.1 \%)$ & $4(25.0 \%)$ & & ND \\
\hline Osteoporosis & $4(8.5 \%)$ & $1(6.7 \%)$ & & ND \\
\hline Diabetes & $1(2.1 \%)$ & $5(33.3 \%)$ & 5.66 (1.84-17.42) & 0.002 \\
\hline Thrombocytopenia & $1(2.1 \%)$ & $3(20.0 \%)$ & & ND \\
\hline \multicolumn{5}{|l|}{ Bacteriology } \\
\hline Pseudomonas aeruginosa & $34(72.3 \%)$ & $8(53.3 \%)$ & $0.46(0.15-1.38)$ & 0.17 \\
\hline MSSA & $22(46.8 \%)$ & $8(53.3 \%)$ & $1.53(0.53-4.42)$ & 0.43 \\
\hline MRSA & 9 (19.1\%) & $1(6.7 \%)$ & $1.17(0.14-9.41)$ & 0.88 \\
\hline Aspergillus & $10(21.3 \%)$ & $2(13.3 \%)$ & $0.88(0.20-3.96)$ & 0.87 \\
\hline Stenotrophomonas & $3(6.4 \%)$ & $1(6.7 \%)$ & & ND \\
\hline Achromobacter & $2(4.3 \%)$ & $1(6.7 \%)$ & & ND \\
\hline \multicolumn{5}{|l|}{ Long-term treatment } \\
\hline RhDNase & $37(78.7 \%)$ & $13(86.7 \%)$ & $2.19(0.28-16.85)$ & 0.45 \\
\hline Inhaled tobramycin & 9 (19.1\%) & $3(20.0 \%)$ & $1.26(0.34-4.71)$ & 0.73 \\
\hline Inhaled colimycin & $12(25.5 \%)$ & $4(25.0 \%)$ & $1.05(0.33-3.36)$ & 0.93 \\
\hline Triazole & $9(19.1 \%)$ & $6(40.0 \%)$ & $4.13(1.41-12.07)$ & 0.009 \\
\hline$>4$ i.v. antibiotic courses & $11(23.9 \%)$ & $3(20.0 \%)$ & $1.08(0.30-3.90)$ & 0.90 \\
\hline $\begin{array}{l}\text { Exacerbations per year } \mathrm{n} \\
\text { (range) }\end{array}$ & $2(0-3)$ & $1(0-3)$ & $1.07(0.85-1.35)$ & 0.57 \\
\hline
\end{tabular}

Data are presented as mean \pm SD or $\mathrm{n}(\%)$, unless otherwise stated. Hazard ratios were calculated using Cox regression models. Multivariate analysis was not performed due to the limited sample size and $\mathrm{MH}$ event number. Bold font indicates statistically significant p-values. Genotype was known for 61 out of the 62 patients. IQR: interquartile range; $\mathrm{FEV}_{1}$ : forced expiratory volume in $1 \mathrm{~s}$; FVC: forced vital capacity; EPI: exocrine pancreatic insufficiency; ABPA: allergic bronchopulmonary aspergillosis; MSSA: methicillin-sensitive Staphylococcus aureus; MRSA: methicillin-resistant S. aureus; RhDNase: recombinant human deoxyribonuclease (dornase alfa); ND: not done. 
cohort, consistent with a report by BARBEN et al. [4], our study was limited by its retrospective design, the small number of patients, the high proportion of excluded patients, and the difficulty in precisely quantifying haemoptysis-associated blood loss. Current practices recommend cessation of recombinant human deoxyribonuclease ( $\mathrm{RhDNase}$ ) aerosols during haemoptysis due to their irritant effects. In our study, RhDNase use was not significantly associated with $\mathrm{MH}$, supporting the findings of a Cochrane review in which haemoptysis was not a significant adverse effect of RhDNase [8].

ABPA infection is a frequent complication in adult patients with $\mathrm{CF}$ and is generally associated with poorer clinical condition. In the present study, ABPA was significantly associated with $\mathrm{MH}$, consistent with previous studies by others [9] and by us [10]. The latter study was a retrospective, case-control, multicentre study in France of 21 adult patients with CF and showed that ABPA and recurrent mild haemoptysis are associated with $\mathrm{MH}$ [10]. However, triazole treatment was prescribed for 15 of the 21 patients with ABPA in the present study and was also significantly associated with $\mathrm{MH}$, suggesting that it did not prevent $\mathrm{MH}$. In our cohort, two of the five patients who had been treated with BAE for the first mild-to-moderate haemoptysis went on to experience $\mathrm{MH}$; although this suggests that BAE was not effective in preventing $\mathrm{MH}$, it is possible that it could have delayed the time to MH. Antonell et al. [11] reported a significant reduction in mild-to-moderate haemoptysis recurrence in eight $\mathrm{CF}$ patients during the 3 years after BAE, although the severity of recurrent mild-to-moderate haemoptysis was not specified. We did not determine whether $\mathrm{MH}$ was associated with haemoptysis treatment with tranexamic acid here because many $\mathrm{CF}$ patients with scant haemoptysis self-medicate with this agent without reporting it to their clinician. It would be of interest to assess the potential protective effect of tranexamic acid on MH occurrence [12].

The prognostic value of nutritional status for $\mathrm{MH}$ occurrence in $\mathrm{CF}$ patients remains unclear. In the present study, we detected a trend towards an association between low BMI and MH occurrence. EFRATI et al. [13] found no significant association between BMI and haemoptysis in 40 CF patients, whereas VIDAL et al. [14] reported that low BMI was significantly associated with increased risk of death or lung transplantation in their study of 57 CF patients with haemoptysis. We note that the small sample size in our study may have precluded detection of some associations [14].

Nevertheless, our findings do concur with other observations by EFRATI et al. [13], including that diseases such as CF-related diabetes, cirrhosis and portal hypertension are more common among patients with a history of haemoptysis than those without (57.5\% versus 5.2\%) [14]. Here, we detected a significant association between CF-related diabetes and $\mathrm{MH}$ occurrence, and four of the five patients with liver cirrhosis experienced $\mathrm{MH}$.

In conclusion, $\mathrm{ABPA}$, metabolic disorders and recurrent mild-to-moderate haemoptysis may predict $\mathrm{MH}$ occurrence in CF patients with at least one mild-to-moderate haemoptysis episode. Whether optimal management of these events would help to reduce the risk of $\mathrm{MH}$ remains to be determined.

\section{Geoffrey Pavaut $^{1}$, Maeva Kyheng ${ }^{2,3}$, Olivier Le Rouzic $\oplus^{1,4,5}$, Thierry Perez ${ }^{1,4,5}$, Benoit Wallaert $\oplus^{1,4,5}$ and Anne Prevotat ${ }^{1,4,5}$}

${ }^{1} \mathrm{CHU}$ Lille, Centre de Ressources et de Compétences de la Mucoviscidose, Service de Pneumologie et Immuno-allergologie, Hôpital Calmette, Lille, France. ${ }^{2}$ Univ. Lille, CHU Lille, ULR 2694 - METRICS: évaluation des technologies de santé et des pratiques médicales, Lille, France. ${ }^{3} \mathrm{CHU}$ Lille, Département de Biostatistiques, Lille, France. ${ }^{4}$ Univ. Lille, Lille, France. ${ }^{5}$ Inserm, CNRS, Institut Pasteur de Lille, U1019 UMR 9017 - CIIL - Center for Infection and Immunity of Lille, Lille, France.

Correspondence: Anne Prevotat, CHU Lille, Centre de Ressources et de Compétences de la Mucoviscidose, Service de Pneumologie et Immuno-allergologie, Hôpital Calmette, Bld Leclercq, Lille 59037, France. E-mail: anne.prevotat@chru-lille.fr

Received: 9 March 2020 | Accepted after revision: 19 July 2020

Acknowledgements: The authors wish to thank Julien Labreuche (Univ. Lille, CHU Lille, ULR 2694 - METRICS: évaluation des technologies de santé et des pratiques médicales, F-59000 Lille, France) for help with the statistical analysis and Anne M. O'Rourke for editing a version of the manuscript.

Author contributions: G. Pavaut, B. Wallaert and A. Prevotat conceived the study. G. Pavaut, A. Prevotat, B. Wallaert and M. Khyeng performed the formal analysis. B. Wallaert and A. Prevotat devised the methodology. G. Pavaut conducted the investigation. G. Pavaut, B. Wallaert and A. Prevotat wrote the original manuscript. All authors reviewed and edited the final manuscript.

Conflict of interest: G. Pavaut has nothing to disclose. M. Kyheng has nothing to disclose. O. Le Rouzic reports personal fees and nonfinancial support from AstraZeneca, Boehringer Ingelheim, Chiesi, Lilly and Novartis, and nonfinancial support from GlaxoSmithKline, MundiPharma, Pfizer, Teva, the Santelys Association, Vertex and Vitalaire, outside the 
submitted work. T. Perez reports nonfinancial support for clinical studies in cystic fibrosis from Physioassist and Antadir outside the submitted work. B. Wallaert reports personal fees and nonfinancial support from Roche and Boehringer Ingelheim, and nonfinancial support from Vitalaire, outside the submitted work. A. Prevotat has nothing to disclose.

\section{References}

1 Goss CH, Burns JL. Exacerbations in cystic fibrosis. 1: Epidemiology and pathogenesis. Thorax 2007; 62: 360-367.

2 Martin C, Hamard C, Kanaan R, et al. Causes of death in French cystic fibrosis patients: the need for improvement in transplantation referral strategies! J Cyst Fibros 2016; 15: 204-212.

3 Thompson V, Mayer-Hamblett N, Kloster M, et al. Risk of hemoptysis in cystic fibrosis clinical trials: a retrospective cohort study. J Cyst Fibros 2015; 14: 632-638.

4 Barben JU, Ditchfield M, Carlin JB, et al. Major haemoptysis in children with cystic fibrosis: a 20-year retrospective study. J Cyst Fibros 2003; 2: 105-111.

5 Flume PA, Mogayzel PJ, Robinson KA, et al. Cystic fibrosis pulmonary guidelines: pulmonary complications: hemoptysis and pneumothorax. Am J Respir Crit Care Med 2010; 182: 298-306.

6 Ibrahim WH. Massive haemoptysis: the definition should be revised. Eur Respir J 2008; 32: 1131-1132.

7 Schidlow DV, Taussig LM, Knowles MR. Cystic Fibrosis Foundation consensus conference report on pulmonary complications of cystic fibrosis. Pediatr Pulmonol 1993; 15: 187-198.

$8 \quad$ Yang C, Montgomery M. Dornase alfa for cystic fibrosis. Cochrane Database Syst Rev 2018; 9: CD001127.

9 Mastella G, Rainisio M, Harms HK, et al. Allergic bronchopulmonary aspergillosis in cystic fibrosis. A European epidemiological study. Epidemiologic Registry of Cystic Fibrosis. Eur Respir J 2000; 16: 464-471.

10 Prevotat A, Bury Q, Hubert D, et al. 239 Outcomes of massive hemoptysis in adult patients with cystic fibrosis J Cyst Fibros 2017; 16: S123-S124.

11 Antonelli M, Midulla F, Tancredi G, et al. Bronchial artery embolization for the management of nonmassive hemoptysis in cystic fibrosis. Chest 2002; 121: 796-801.

12 Al-Samkari H, Shin K, Cardoni L, et al. Antifibrinolytic agents for hemoptysis management in adults with cystic fibrosis. Chest 2019; 155: 1226-1233.

13 Efrati O, Harash O, Rivlin J, et al. Hemoptysis in Israeli CF patients-prevalence, treatment, and clinical characteristics. J Cyst Fibros 2008; 7: 301-306.

14 Vidal V, Therasse E, Berthiaume Y, et al. Bronchial artery embolization in adults with cystic fibrosis: impact on the clinical course and survival. J Vasc Interv Radiol 2006; 17: 953-958. 